\title{
FACTORES PRONÓSTICOS DEL CARCINOMA DUCTAL IN SITU DE MAMA
}

\author{
Alejandra Martínez RM., Iria Aparicio R., Ginés Hernández C., María del Mar Muñoz M., \\ Javier de Santiago G.
}

Servicio de Ginecología y Obstetricia, Hospital Universitario La Paz, Madrid, España.

\section{RESUMEN}

Objetivo: El objetivo primario del estudio es evaluar el impacto del índice pronóstico de Van Nuys (VNPI) en la recurrencia del carcinoma ductal in situ de mama (CDIS). Entre los objetivos secundarios se incluye la valoración de otros parámetros histológicos: el tamaño, el grado nuclear, la necrosis, la supervivencia libre de enfermedad y la supervivencia global. Método: Se analizaron los casos con diagnóstico de CDIS entre enero de 1995 a diciembre de 2000, de la base de datos de la Unidad de Mama del Hospital Universitario La Paz, Madrid, España. Se han recogido los hallazgos mamográficos, histológicos, así como la evolución de las pacientes. Resultados: Se diagnosticaron 95 CDIS en el periodo estudiado, de los que sólo se incluyeron 79 casos en el análisis estadístico. EI CDIS representa el 7,1\% de todos los cánceres de mama en nuestro centro. La presencia de microcalcificaciones en la mamografía de cribado es el hallazgo diagnóstico mas frecuente. Ninguna de las variables clínicas analizadas se asoció a una mayor tasa de recaídas. Se asoció significativamente $(p<0,05)$ a una mayor tasa de recurrencias el tamaño tumoral, el alto grado y la necrosis, así como el VNPI. Conclusiones: EI CDIS tiene un comportamiento muy variable y ha de considerarse como entidades aisladas más que como una única enfermedad. Los parámetros biológicos e histológicos, principalmente el VNPI, podrían ayudar a individualizar el tratamiento.

\section{PALABRAS CLAVE: Carcinoma ductal in situ de mama, índice pronóstico de Van Nuys}

\section{SUMMARY}

Objective: The primary endpoint of our study was to evaluate the impact of Van Nuys Prognostic Index (VNPI) on recurrence of the breast ductal carcinoma in situ (DCIS). Secondary endpoints included other histologic features: size, nuclear grade, necrosis and architectural pattern, disease-free survival and overall survival. Methods: Database of the breast unit was searched for patients with DCIS diagnose between January 1995 and December 2000. Clinical data, mammographic findings, pathological features, outcome and prognosis were studied. Results: Ninty five DCIS were detected at our center between 1995-2000, but only 79 patients were included in the statistical analysis. The incidence was $7.1 \%$ over the total number of breast cancers. Diagnose was made by a screening mammography in the majority of cases, and presence of microcalcifications was the most frequent mammographic finding. None of the clinical variables was associated to bigger rates of relapse. Tumour size, high grade and necrosis and VNPI had a significant association with recurrence. Conclusions: Disease outcomes are very diverse, and it is necessary to consider DCIS as different entities, more than as an isolated disease. Biologic and histological factors, mainly VNPI, allow to stratify patients according to their risk and help to individualize treatment.

\section{KEY WORDS: Ductal carcinoma in situ of the breast, Van Nuys prognostic index}




\section{INTRODUCCIÓN}

El carcinoma ductal in situ (CDIS) de mama se ha convertido en una entidad frecuente con la sistematización de la mamografía de cribado. En la actualidad entre un $15-30 \%$ de los cánceres de mama son detectados en la primera mamografía (1). El CDIS es una enfermedad muy heterogénea, lo que se refleja en su forma de presentación, características histolopatológicas y radiológicas, expresión de marcadores biológicos y comportamiento clínico (2). Se han identificado factores de riesgo implicados en las recaídas y en la evolución a carcinoma invasor, tales como edad menor a 45 años, presencia de márgenes positivos, factores histológicos (pobre diferenciación nuclear y aparición de comedonecrosis) y alteraciones genéticas (3-9).

La clasificación histológica como único parámetro para determinar el tratamiento resulta inapropiada para algunos autores y, aunque los parámetros histológicos tienen un valor pronóstico indudable, la gran variabilidad interobservador limita su uso (2).

Las variables histológicas de mayor importancia en el CDIS son: grado nuclear (alto, intermedio o bajo grado), necrosis (comedonecrosis o punctata) y patrón arquitectural (comedo, cribiforme, papilar, micropapilar y sólido) $(2,10,11)$. El índice pronóstico de Van Nuys (VNPI) se elaboró para ayudar a elegir el tipo de terapia más adecuada (12). Combina tres factores de riesgo para la recurrencia local: tamaño tumoral, espesor de los márgenes y clasificación patológica. En 2001, la Universidad de California del Sur definió la edad como un parámetro pronóstico aislado, añadiendo esta cuarta variable al VNPI (VNPIm) y le dieron la misma importancia que a las otras tres (13).

El objetivo primario del estudio es evaluar, el impacto del VNPI en las recaídas. El análisis secundario incluye el estudio de otros parámetros histológicos (tamaño tumoral, grado nuclear, necrosis y patrón arquitectural) y su asociación a la tasa de recidivas; así como el cálculo de la supervivencia libre de enfermedad y de la supervivencia global, en 95 casos de CDIS atendidos en la Unidad de Mama del Hospital Universitario La Paz, Madrid, España.

\section{MATERIAL Y MÉTODOS}

Estudio retrospectivo de las pacientes con diagnóstico de CDIS de mama desde enero de 1995 hasta diciembre de 2000 en el Hospital Universitario La Paz, Madrid, España.

Pacientes: Se identificaron 95 casos de CDIS entre 1995-2000. Se excluyeron las pacientes con carcinoma invasor o microinvasor, CDIS con afec- tación de la epidermis, CDIS previo o pacientes con afectación ganglionar. Finalmente, se incluyeron un total de 79 pacientes en el análisis estadístico. Los datos recogidos fueron: 1 . edad, 2 . estado menopáusico, 3. método de detección, 4. hallazgos mamográficos, 5. tratamiento: tipo de cirugía (tumorectomía vs mastectomía), radioterapia, quimioterapia, uso de tamoxifeno, 6. histología: tamaño tumoral, arquitectura celular (comedocarcinoma, cribiforme, micropapilar, sólido, papilar, formas mixtas), espesor del margen quirúrgico, grado nuclear, VNPIm, estado de receptores hormonales de estrógeno (RE) y progesterona (RP) (definidos como positivos por un nivel de $10 \mathrm{fmol} / \mathrm{mg}$ o un resultado positivo en el análisis inmunohistoquímico). Los datos histológicos se recogieron siguiendo las recomendaciones de la Conferencia de Consenso sobre la clasificación del CDIS de 1998. De las recaídas se recogieron los siguientes datos: 1. localización (local, ipsilateral o contralateral, carcinoma invasor con metástasis a distancia), 2. parámetros histológicos.

Análisis estadístico: Realizado con SYSTAT, versión 10.0 (SPSS Inc.m Chicago, IL). En el análisis descriptivo, se usó mediana, media, rango y desviación estándar para variables cuantitativas y tablas de frecuencias para variables categóricas. Se consideró un $\mathrm{p}<0,05$ como diferencia estadísticamente significativa. Se utilizó el test de Chi-cuadrado para variables categóricas. Las variables continuas se analizaron con la prueba no paramétrica $U$ de Mann-Whitney. Las curvas de intervalo libre de enfermedad y de supervivencia global se calcularon según análisis de Kaplan-Meier.

\section{RESULTADOS}

Las carácterísticas clínicas, mamográficas, tipo de cirugía y tratamiento han sido similares en el grupo libre de enfermedad y en el que presentó recaída (Tabla I). En el análisis de las variables histológicas hubo diferencias estadísticamente significativas para alto grado y necrosis tumoral $(p<0,02)$, tamaño $(p<0,01)$ y VNPI $(p<0,002)$ (Tabla II).

Ocho pacientes $(10,1 \%)$ presentaron recaída, que fue local en el $75 \%$ de los casos (en forma de CDIS o de carcinoma invasor), y en forma de carcinoma infiltrante con metástasis a distancia en el $25 \%$ restante (Figura 1 ). El tamaño tumoral de las recaídas locales presentó una mediana de $15 \mathrm{~mm}$ y una media de 17,86 mm (rango: 11-35 mm).

En las recaídas locales el tratamiento quirúrgico fue tumorectomía con linfadenectomía en un $50 \%$, mastectomía más linfadenectomía en un 37,5\%, y en el $12,5 \%$ restante ( 1 paciente) se realizó una resección local, ya que se trataba de una recidiva a 
Tabla I

CARACTERÍSTICAS CLÍNICAS

\begin{tabular}{lccc}
\hline $\begin{array}{l}\text { CARACTERÍSTICAS } \\
\text { CLÍNICAS }\end{array}$ & L.E & Recaídas & Valor $p$ \\
\hline $\begin{array}{l}\text { Edad (años) } \\
\text { Mediana }\end{array}$ & 59 & 52,50 & \\
Media & 56,59 & 50,63 & 0,22 \\
Rango & $23-78$ & $29-68$ & \\
Estado hormonal & & & \\
$\quad$ (\%) & & & \\
Premenopáusica & 26,8 & 37,5 & \\
Perimenopáusica & 5,6 & 25 & 0,08 \\
Postmenopáusica & 67,6 & 37,5 & \\
Síntomas & & & \\
$\quad$ (\%) & & & \\
Presentes & 22,5 & 25 & 0,58 \\
Ausentes & 77,5 & 75 & 0,84 \\
\hline Microcalcificaciones & & & \\
$\quad$ (\%) & 70,4 & 87,5 & \\
\hline
\end{tabular}

L.E: Seguimiento libre de enfermedad

nivel de la cicatriz de mastectomía previa con metástasis óseas y en SNC. El otro caso de recidiva infiltrante con metástasis a distancia fue una paciente con una masiva afectación ganglionar (23 de 26 ganglios analizados), en la que posteriormente se evidenció enfermedad diseminada. Ambos casos habían sido tratados inicialmente mediante mastectomía.

Permanecen libres de enfermedad el 93\% a los 5 años y el $89 \%$ a los 7 años (Figura 2). En cuanto a la supervivencia global, el $89 \%$ sigue viva a los 7 años del diagnóstico (Figura 3).

\section{DISCUSIÓN}

La incidencia de CDIS asintomático en nuestra serie representa el $77,2 \%$ de los casos. Esta cifra ha aumentado a lo largo de los años a raíz de la introducción del cribado sistemático de mama, lo que se refleja en el estudio de Meijnen y cols (14), con un aumento en la incidencia de CDIS asintomático del 47 al $77 \%$ desde 1986 a 2002.

Ninguna de las variables clínicas se asoció a un mayor número de recaídas. La edad es menor en el grupo de las recaídas (media 56,59 vs 50,63 años), si bien no alcanzó significación estadística.

Sin embargo, hay un $39,4 \%$ de pacientes meno-
Tabla II ANATOMÍA PATOLÓGICA

\begin{tabular}{llll}
\hline \multicolumn{1}{c}{ VARIABLES } & L.E & Recaídas & Valor $\mathrm{p}$ \\
\hline Patrón arquitectural (\%) & & & \\
Comedocarcinoma & 2,8 & - & \\
Cribiforme & 29,6 & 25 & \\
Micropapilar & 5,6 & 12,5 & \\
Papilar & 1,4 & 12,5 & 0,1 \\
Sólido & 11,3 & 25 & \\
Mixto cribiforme-micropapilar & 53,5 & 12,5 & \\
Mixto sólido-cribiforme & 19,7 & - & \\
\hline Necrosis (\%) & 67,2 & 71,4 & 1,0 \\
\hline Grado nuclear (\%) & & & \\
Grado I & 35,3 & - & 0,075 \\
Grado II & 32,4 & 28,6 & \\
Grado III & 32,4 & 71,4 & \\
\hline Alto grado más necrosis (\%) & 26,8 & 71,4 & 0,02 \\
\hline Tamaño (mm) & & & \\
Mediana & 12 & 20 & 0,01 \\
Media & 15,3 & 29,7 & \\
Rango & $1,5-50$ & $11-56$ & \\
\hline Margen quirúrgico (\%) & & &
\end{tabular}

Margen quirúrgico (\%)

\begin{tabular}{llcc} 
0-2 mm & 4,5 & - & 0,2 \\
$3-9 \mathrm{~mm}$ & 22,8 & 37,5 & \\
$\geq 10 \mathrm{~mm}$ & 72,7 & 62,5 & \\
\hline VPNI (\%) & & & \\
Bajo & 8,3 & - & 0,002 \\
Intermedio & 76,7 & 28,6 & \\
Alto & 15 & 71,4 & \\
\hline Receptores hormonales (\%) & & & \\
RE + & 55,6 & 50 & 1,0 \\
RP + & 44,4 & 50 & 1,0 \\
\hline
\end{tabular}

LE: Libre de enfermedad

res de 55 años en el grupo con seguimiento libre de enfermedad, cifra que aumenta al $75 \%(p<0,06)$, diferencia no significativa, entre las pacientes que han sufrido una recaída. Muchos autores identifican una edad menor a 45 años como un factor pronóstico adverso, que potencia la recidiva ipsilateral $(1,7)$.

Este subgrupo de pacientes parece tener factores histológicos más desfavorables como peor grado de diferenciación celular, mayor proporción de necrosis y mayor afectación de márgenes quirúrgi- 


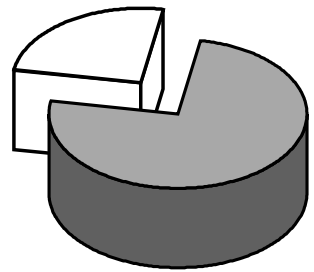

$\square$ CDIS $\square$ CDInfiltrante

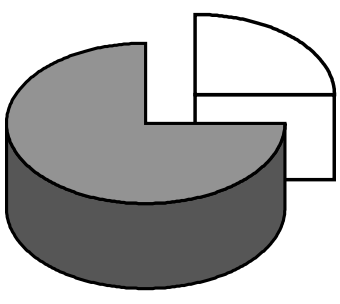

$\square$ Grado II $\square$ Grado III
Figura 1. Histología de las recaídas

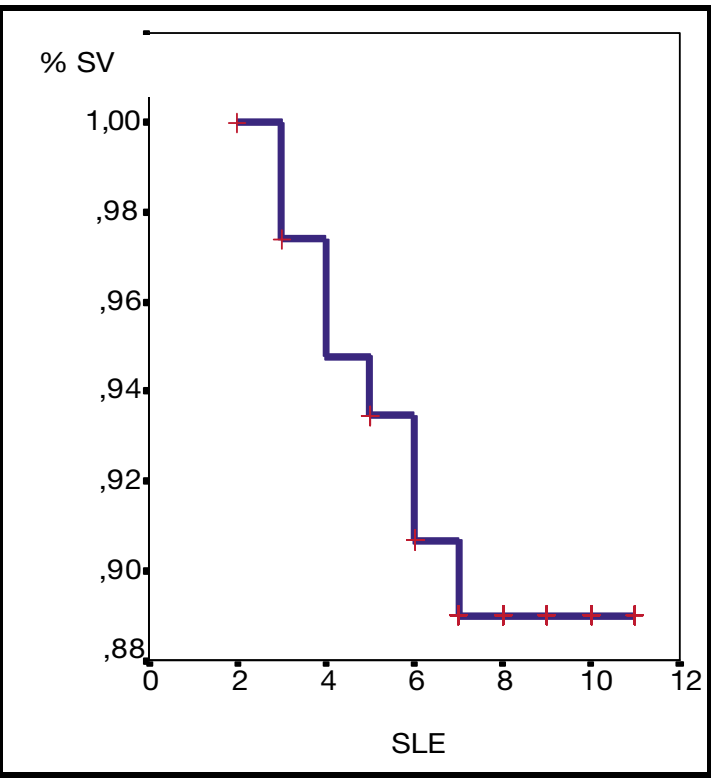

Figura 2. Suprvivencia libre de enfermedad

cos, probablemente debido a un menor tamaño en la biopsia inicial (15).

Arquitectura celular: No encontramos diferencias en cuanto a los distintos tipos histológicos de tumor. Aunque para la mayoría de los autores el subtipo comedocarcinoma confiere peor pronóstico $(2,16,17)$, en nuestra serie, el escaso número de recaídas y la superposición de distintos patrones arquitecturales dificultan la interpretación de los datos. Además, debido a la interpretación subjetiva del patrón arquitectural y de la coexistencia de varios patrones, distintos patólogos pueden no coincidir en el tipo de CDIS (18).

Grado nuclear: Un pobre grado de diferenciación tumoral es considerado por muchos autores como el factor pronóstico histológico más importante ya que se asocia a una mayor tasa de recaídas y a un menor intervalo de tiempo hasta la recaída $(3,8,19$ 21). A pesar de no alcanzar significación estadística $(p<0,075)$ en nuestra serie, el $71,4 \%$ de las pacientes que tienen una recidiva presentan un grado nuclear tipo 3, frente al 32,4\% de las que están libres de enfermedad, dato que coincide con la literatura.

El grado 3 no sólo se ha relacionado con las recidivas locales $(3,8,19-21)$, sino también con un menor intervalo libre de enfermedad (8), diseminación a distancia y muerte (7). En nuestra serie, las dos únicas pacientes con carcinoma infiltrante y metástasis a distancia presentaban un grado nuclear 3 al diagnóstico. Se ha asociado un alto grado nuclear más necrosis con un riesgo extremo de recurren-

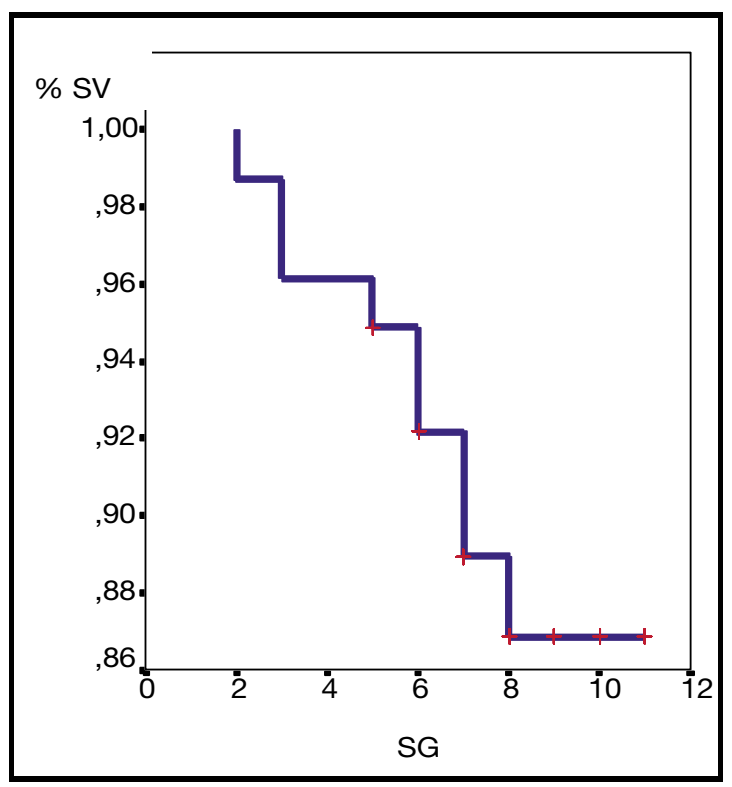

Figura 3. Supervivencia global.

cia $(22,23)$, dato que coincide con nuestra serie. El $26,9 \%$ de las pacientes libres de enfermedad cumplen ambas características, frente a un $71,4 \%$ en el grupo que presentó una recaída $(p<0,002)$.

Tamaño tumoral: El tamaño tumoral al diagnóstico influye en la evolución de la paciente $(2,8)$, se ha evidenciado que existe una mayor tasa de recaídas y un menor intervalo de tiempo hasta la recidiva en pacientes con un tamaño tumoral elevado (8). También nosotros encontramos tal diferencia, con una mayor tasa de recaídas en el subgrupo de pacientes con tamaño tumoral superior $(p<0,01)$. Esta mayor tasa de recaídas podría ser debida a una asociación con multifocalidad y con focos de microinfiltración no detectados.

Margen quirúrgico: El margen quirúrgico es un dato clave en la tasa de recurrencias, y para muchos autores constituye el factor más determinante en el pronóstico $(1,4,7,23-25)$. Es importante tener 
en cuenta que pueden existir dificultades técnicas en la valoración histológica y radiológica del espesor del margen quirúrgico $(26,27)$. La recurrencia local tras cirugía conservadora se debe en muchos casos a una cirugía inadecuada con persistencia de enfermedad residual. Es por ello, que la mayoría de las recurrencias locales tras cirugía conservadora en el CDIS aparecen en el mismo foco o muy cerca de la lesión primaria (25). Un margen quirúrgico amplio, superior a $10 \mathrm{~mm}$, permite por ello un excelente control local.

VNPIm: En un intento de predecir las recurrencias en el CDIS algunos autores han hallado correlación significativa con el VNPI $(21,23,28-30)$. El desarrollo del VNPIm ha permitido establecer una gradación de riesgo para ayudar a decidir entre una terapia u otra. En los CDIS con puntuaciones bajas se podría hacer una excisión aislada, en los que presentan puntuaciones intermedias habría que añadir radioterapia $(\mathrm{Rt})$ o bien hacer una reexcisión de los márgenes si son $<10 \mathrm{~mm}$, y en los CDIS con puntuaciones altas habría que considerar además de la Rt, la mastectomía con reconstrucción inmediata, si es posible realizarla, o la resección si fuese técnicamente viable (28). En nuestra serie, el $71,4 \%$ de las pacientes en el grupo de las recaídas presentaron un VNPIm alto, mientras que no hubo ninguna entre las pacientes con un VNPIm bajo. Sin embargo, sólo se practicó mastectomía al 33,3\% de las pacientes con VNPIm alto. Aunque hay diversos estudios que muestran una menor tasa de recurrencia en las pacientes con mastectomía (alrededor del 1\%), la falta de diferencia en las tasas de supervivencia respecto a la cirugía conservadora, hace que se opte cada vez más por conservar la mama (25). En la actualidad, la mastectomía está quedando relegada a lesiones grandes, tumores multicéntricos, persistencia de un foco de calcificaciones tras la cirugía, afectación del margen quirúrgico, otras contraindicaciones para conservar la mama, o por elección personal de la paciente $(4,20)$.

Radioterapia $(R t)$ : En nuestra serie se han tratado con tumorectomía y radioterapia un $38,2 \%$ de las pacientes, y no se han encontrado diferencias entre estas pacientes frente a las tratadas sin Rt. Esto probablemente debido al escaso número de pacientes (3 pacientes) que se han tratado con tumorectomía aislada. En los tres estudios prospectivos más relevantes que comparan tumorectomía más $R t$ versus tumorectomía aislada $(1,9,16,17,31)$, aparecen diferencias a favor de la Rt. En el estudio NASBP B-17 $(16,17,31)$ la incidencia de recurrencia local es del $26,8 \%$ en el grupo tratado con tumorectomía aislada frente a un $12,1 \%$ en el grupo con Rt, y la de recidiva de CDIS es del $13,4 \%$ y del $8,2 \%$ al añadir Rt. Sin embargo, el espesor del margen quirúrgico y la mamografía post-cirugía no se determinaron, lo que limita la validez del estudio al desconocer si la excisión tumoral fue completa. En el EORTC (1), el intervalo libre de enfermedad es significativamente mayor en el grupo de Rt. Obtienen cifras de recurrencia local a los 4 años de $16 \%$ en el grupo tratado con tumorectomía aislada frente a un $9 \%$ en el grupo con Rt. El UK CCCR (9) también encontró una disminución en la tasa de recurrencia local en el grupo de pacientes tratadas con Rt superior al 50\%. Sin embargo, en ninguno de los tres estudios aparecen mejoras en la supervivencia, y ninguno de los tres distingue cuál es el subgrupo de pacientes que realmente se beneficia de la Rt. Las lesiones con un margen igual o superior a $10 \mathrm{~mm}$ tienen una supervivencia libre de enfermedad muy buena y el añadir $\mathrm{Rt}$ al tratamiento no reduciría las tasas de recurrencia local (7). En nuestra serie no se encontró una asociación entre el uso de Rt y la tasa de recidiva, pero la mayoría de los autores concluyen que la radioterapia tras cirugía conservadora disminuye la probabilidad de recaída. Sin embargo, tumores bien diferenciados con márgenes superiores a $10 \mathrm{~mm}$ y con un VNPI bajo, probablemente no se beneficiarían de tal efecto $(1,21,23,25)$. Además del mayor costo de la Rt, esta se acompaña de efectos secundarios en un pequeño grupo de pacientes $y$, si se usa en el CDIS, impide de nuevo su uso en una eventual recurrencia invasiva (7). Por ello, sería importante examinar qué subgrupo de pacientes es el que se beneficiaría más de la Rt. Así, en las pacientes de bajo riesgo podría plantearse la tumorectomía aislada $(20,33)$.

Tamoxifeno (TMX): El número de pacientes tratadas con TMX es similar en ambos grupos. El estudio prospectivo NASBP-B24 $(4,31)$ a 8 años, muestra una disminución en el riesgo de cáncer de mama ipsilateral si se añade TMX. Sin embargo, no se intentó identificar si algún subgrupo de pacientes no se beneficiaría del TMX, ni se evaluó la sobreexpresión del c-erbB-2, y el papel del TMX sin la administración de radioterapia es especulativo. El estudio UK CCCR (9), no encuentra diferencias en el uso de TMX y la tasa de recaídas. Ninguno de los dos estudios evidencia diferencias en la supervivencia.

\section{CONCLUSIONES}

EI CDIS es una enfermedad muy heterogénea con un comportamiento clínico diverso y hay que considerar la enfermedad como distintos tipos de lesiones, más que como una entidad aislada. En nuestra cohorte de pacientes, los parámetros que 
se han asociado con un peor pronóstico son: el tamaño tumoral, el grado nuclear más necrosis y el VNPIm.

Los factores histológicos, principalmente el VN$\mathrm{PIm}$, pueden servir de guía para estratificar a las pacientes según el riesgo y ayudarnos en las decisiones terapéuticas. Se requieren estudios prospectivos y randomizados amplios para determinar la importancia de los factores histológicos en el pronóstico de forma que se pueda individualizar y optimizar el tratamiento.

\section{BIBLIOGRAFÍA}

1. Julien JP, Bijker N, Fentiman IS, Peterse JL, Delledonne $V$, Rouanet $P$, et al. Radiotherapy in breast conserving treatment for ductal carcinoma in situ: first results of the EORTC randomised phase III trial 10853. EORTC Breast Cancer Cooperative Group and EORTC Radiotherapy Group. Lancet 2000;355:528-33.

2. Jaffer S, Bleiweiss IJ. Histologic classification of ductal carcinoma in situ. Microsc Res Tech 2002;59(2):92101.

3. Van de Vijver MJ. Biological variables and prognosis of DCIS. Breast 2005;14(6):509-19.

4. Fisher B, Dignam J, Wolmark N, Wickerham DL, Fisher ER, Mamounas $\mathrm{E}$, et al. Tamoxifen in treatment of intraductal breast cancer: National Surgical Adjuvant Breast and Bowel Project B-24 randomised controlled trial. Lancet 1999;353(9169):1993-2000.

5. Fisher ER, Costantino J, Fisher B, Palekar AS, Redmond C, Mamounas E. Pathologic findings from the National Surgical Adjuvant Breast Project (NSABP) Protocol B-17. Intraductal carcinoma (ductal carcinoma in situ). The National Surgical Adjuvant Breast and Bowel Project Collaborating Investigators. Cancer 1995;75(6):1310-9.

6. Fisher ER. Pathobiological considerations relating to the treatment of intraductal carcinoma (ductal carcinoma in situ) of the breast. CA Cancer $\mathrm{J}$ Clin 1997;47(1):52-64.

7. Vargas C, Kestin L, Go N, Krauss D, Chen P, Goldstein $\mathrm{N}$, et al. Factors associated with local recurrence and cause-specific survival in patients with ductal carcino$\mathrm{ma}$ in situ of the breast treated with breast-conserving therapy or mastectomy. Int J Radiat Oncol Biol Phys 2005;63(5):1514-21.

8. Cornfield DB, Palazzo JP, Schwartz GF, Goonewardene SA, Kovatich AJ, Chervoneva I, et al. The prognostic significance of multiple morphologic features and biologic markers in ductal carcinoma in situ of the breast: a study of a large cohort of patients treated with surgery alone. Cancer 2004;100(11):2317-27.

9. George W. UK CCR Trial. Lancet 2003;362:95-102.

10. Chitemerere M, Andersen T.I, Holm R, Karlsen F, Borressen AL, Nesland JM. TP53 alterations in atypical ductal hyperplasia and ductal carcinoma in situ of the breast. Breast Cancer Res Treat 1996;41:103-9.

11. Consensus Conference on the classification of ductal carcinoma in situ. The Consensus Conference Com- mittee. Cancer 1997;80(9):1798-802.

12. Silverstein MJ, Lagios MD, Craig PH, Waisman JR, Lewinsky BS, Colburn WJ, Poller DN. A prognostic index for ductal carcinoma in situ of the breast. Cancer 1996;77(11):2267-74.

13. Silverstein MJ. The University of Southern California/ Van Nuys prognostic index for ductal carcinoma in situ of the breast. Am J Surg 2003;186(4):337-43.

14. Meijnen P, Peterse JL, Oldenburg HS, Woerdeman LA, Rutgers EJ. Changing patterns in diagnosis of ductal carcinoma in situ of the breast. EJSO 2005;31:833-9.

15. Goldstein NS, Vicini FA, Kestin LL, Thomas M. Differences in the pathologic features of ductal carcinoma in situ of the breast based on patient age. Cancer 2000;88(11):2553-60.

16. Fisher B, Dignam J, Wolmark N, Mamounas E, Costantino J, Poller $\mathrm{W}$, et al. Lumpectomy and radiation therapy for the treatment of intraductal breast cancer: findings from National Surgical Adjuvant Breast and Bowel Project B-17. J Clin Oncol 1998;16(2):441-52.

17. Fisher ER, Land SR, Saad RS, Fisher B, Wickerham DL, Wang M, Pathologic variables predictive of breast events in patients with ductal carcinoma in situ. Am J Clin Pathol 2007;128(1):86-91.

18. Rosai J. Borderline epithelial lesions of the breast. Am J Surg Pathol 1991;15(3):209-21.

19. Roka S, Rudas M, Taucher S, Dubsky P, BachleitnerHofmann T, Kandioler D, et al. High nuclear grade and negative estrogen receptor are significant risk factors for recurrence in DCIS. EJSO 2004; 30: 243-7.

20. Leonard GD, Swain SM. Ductal carcinoma in situ, complexities and challenges. J Natl Cancer Inst 2004;96(12):906-20.

21. Silverstein MJ, Lagios MD, Craig PH, Waisman JR, Lewinsky BS, Colburn WJ, et al. A prognostic index for ductal carcinoma in situ of the breast. Cancer 1996;77(11):2267-74.

22. Solin LJ, Kurtz J, Fourquet A, Amalric R, Recht A, Bornstein BA, et al. Fifteen-year results of breast-conserving surgery and definitive breast irradiation for the treatment of ductal carcinoma in situ of the breast. $\mathrm{J}$ Clin Oncol 1996;14(3):754-63.

23. Silverstein MJ, Lagios MD, Groshen S, Waisman JR, Lewinsky BS, Martino S, et al. The influence of margin width on local control of ductal carcinoma in situ of the breast. N Engl J Med 1999;340(19):1455-61.

24. Ceilley E, Jagsi R, Goldberg S, Kachnic L, Powell S, Taghian A. The management of ductal carcinoma in situ in North America and Europe. Results of a survey. Cancer 2004;101(9):1958-67.

25. Silverstein MJ, Buchanan C. Ductal carcinoma in situ: USC/Van Nuys Prognostic Index and the impact of margin status. Breast 2003;12(6):457-71.

26. Shnitt SJ, Harris JR, Smith BL. Developing a prognostic index for ductal carcinoma in situ of the breast. Are we there yet? Cancer 1996;77:2189-92.

27. Shoker BS, Sloane PJ. DCIS grading schemes and clinical implications. Histopathology 1999;35:393-400.

28. Silverstein MJ. The University of Southern California/ Van Nuys prognostic index for ductal carcinoma in situ of the breast. Am J Surg 2003;186(4):337-43.

29. Badve SA, Hern R, Ward AM, Millis RR, Pinder SE, 
Ellios IO, et al. A Long term study comparative study of the ability of 5 classifications of ductal carcinoma in situ of the breast to predict local recurrence after surgical excision. Hum Pathol 1998;29:915-23.

30. Silverstein MJ, Poller DN, Waisman JR, Colburn W, Barth A, Gierson ED, et al. Prognostic classification of breast ductal carcinoma -in-situ. Lancet 1995;345:1154-7.

31. Fisher B, Land S, Mamounas E, Dignam J, Fisher ER, Wolmark N. Prevention of invasive breast cancer in women with ductal carcinoma in situ: an update of the national surgical adjuvant breast and bowel project experience. Semin Oncol 2001;28(4):400-18.
32. Houghton J, George WD, Cuzick J, Duggan C, Fentiman IS, Spittle M. UK Coordinating Committee on Cancer Research; Ductal carcinoma in situ Working Party; DCIS trialists in the UK, Australia, and New Zealand. Radiotherapy and tamoxifen in women with completely excised ductal carcinoma in situ of the breast in the UK, Australia, and New Zealand: randomised controlled trial. Lancet 2003;362(9378):95-102.

33. Schwartz GF, Solin LJ, Olivotto IA, Ernster VL. Consensus Conference on the treatment of in situ ductal carcinoma of the breast. Cancer 2000;88:946-54. 